\author{
Uwe Scheffler
}

\title{
EVENTS AS SHADOWY ENTITIES
}

\section{CONTENTS}

\author{
1. Introduction \\ 2. Event terms \\ 3. Events \\ 4. Parts \\ 5. Fictions \\ 6. Negative events
}




\section{Introduction}

The general background of this paper* is causal theory. Events are involved in causal theory in a very natural way: People want to assert causal sentences and in at least some of these sentences terms occur describing events. After Brand [1], Davidson [3], Kim [6], Lewis [7], Lombard [8], van Benthem [13], van INWAGEN [14], and a lot of highly interesting and important other philosophers there is still no common understanding of events. Even all of the following statements which will be presupposed in this paper may be debatable:

1. A useful distinction is that between event tokens and event types. Event tokens are particulars occurring therefore at a concrete and singular position in space and time, whereas event types are universals.

2. An event is something that happens - if it happens - in the world of empirical things and not, for example, in the field of mathematics or moral categories.

3. Events are usually given by description, including a descriptive sentence about what is the case and about the spatiotemporal region in which it is the case.

4. There are sometimes different descriptions of the same event.

5. Events may be complex: they may have spatiotemporal parts of the same kind or may even consist of two or more other events.

In order to clarify the sense of the above mentioned assumptions consider the following examples:

\section{Example 1.}

1. The event, that Brutus killed Caesar at the Ides of March in Rome.

2. The event, that Brutus stabbed Caesar at the Ides of March in Rome.

3. The event, that a tyrant was killed.

4. The event, that Good overcomes Evil.

The first two of the examples stand for tokens, while the third stands for a type. The first two examples describe the same event, which is - under

* This paper belongs to a larger common project on causal logic of MAX URCHS and me. I am indebted to him for various valuable discussions about the topic of this paper.

A first version of the paper was supported by a grant of the Fritz Thyssen Stiftung. 
a certain description - complex: it consists of preparing the assassination, stabbing several times, and waiting for the death of the victim. The last group of words does not describe an event at all, but rather a doctrine or a wish.

The paper contains an non decisive but convincing argument for linguistic conventionalism with respect to events, the idea, that events are language-dependent entities. VAN BENTHEM expresses this view by

It is obvious that events are linguistic constructions. Experience is a continuous movie, which humans cut into manageable slices using their language. ([13], p. 113)

If this is right, two conclusions should be drawn: What events human beings get depends on the language actually used and on human aims and intentions; the ontological question of what events are changes to the terminological question of how to manage event terms. On the other hand it is possible to take part in events, to see events. Hence, even if events are not ontologically independent, it is necessary to show on which entities they are supervenient.

The argument goes as follows: The starting point is a canonical construction of event terms from sentences. The next step consists in claiming a minimal and reasonable ontological thesis about when events exist. It will be shown that even from quite weak and natural assumptions the existence of infinitely many very strange events follows. After discussing some improvements concerning parts of events and negative events the thesis will be: Only the introduction of some kind of epistemological involvement avoids the two extreme consequences: an overcrowded ontology with really shadowy entities, or the lack of events in the ontology at all.

\section{Event terms}

Most of our sentences are understandable only in the context of a conversation. Often this context contains especially the reference to a spatiotemporal region or to concrete objects, to which general terms refer. Consider the following sentences:

\section{Example 2.}

1. It never rains in Southern California.

2. If Adam is older than his brother, the brother is younger than Adam.

3. Some conspirators killed Caesar. 
The first sentence does not need any clarification about the spatiotemporal region, to which it refers. Concerning time, never works like a quantifier ranging over variables for time points or intervals, and Southern California is a name of the place, about which the statement was made. Of course, speaking about Southern California or being there, one can claim "It never rains", and express the same information as in (1.). In this case the context of utterance gives us the possibility of adding here or in Southern California in order to make the sentence verifiable or falsifiable. The second sentence does not contain any linguistic material referring to spatiotemporal regions. Nevertheless, it is understandable outside of any further context, because its truth is grounded only on the meaning of the predicates being older and being younger. In all such cases of analytical or logical truth or falsity it is easy to imagine a universal quantifier over spatiotemporal regions at the beginning of the sentences: they hold or do not hold, everytime and everywhere. The interesting point of the third example is the occurrence of some with respect to conspirators. If a sentence contains unquantified general subject terms, it is usually either treated as its universal closure, or it is ambiguous and needs for further information from the context. The use of quantified general terms and singular terms avoids this kind of context dependence, as is understandable from the third sentence.

It is possible to map all context dependent sentences (with respect to spatiotemporal regions and general terms) into independent sentences:

Regard all sentences as completed by constants or bound variables for spatiotemporal regions as far as they are (in this respect) understandable by themselve. These sentences are universal as opposed to local. ${ }^{1}$

Regard all sentences containing free general terms as completed by special quantifiers over general terms; the resulting sentences are singular in contrary to general sentences. ${ }^{2}$

Spatiotemporal regions and general subject terms will play an decisive role in the construction of event types. It is therefore necessary to use a language, which includes at least the following parts:

variables for spatiotemporal regions: $l, l^{1}, l^{2}, \ldots$; constants for spatiotemporal regions: $t, t^{1}, t^{2}, \ldots$; quantifiers for spatiotemporal regions: $\forall, \exists$;

\footnotetext{
1 Some more detailed information about the introduced differentiation can be found in [10], pp. 496f.

2 The choice of this terminology will become understandable after the introduction of event terms.
} 
singular subject terms: $v, v^{1}, v^{2}, \ldots$;

general subject terms: $w, w^{1}, w^{2}, \ldots$;

quantifiers for general subject terms: $\Sigma, \Pi$;

predicate terms $P, P^{1}, P^{2}, Q, Q^{1}, Q^{2}, \ldots$;

truth-functional operators: $\neg, \vee, \wedge$

the term forming operator: $t^{\star}$, forming names of sentences or names of terms from sentences or terms respectively;

the constant predicate of meaning inclusion between terms $\rightarrow$;

the constant predicates of positive and negative predication $\leftarrow$ and $\nleftarrow$.

Intuitively meaning inclusion means that everything, that is named by the first term, is also named by the second term; it holds either between subject terms or between predicate terms, but not between a subject and a predicate term. Positive predication means that an object has a property (or objects are standing in a relation), negative predication means that the property (or relation) is denied of the object (the objects). Obviously there is a definable third kind of predication: It is not true that the object has the property and it is not true that the property is denied; this is uncertainty predication ${ }^{3}$ :

\section{Example 3.}

$$
\begin{aligned}
& t^{\star}(\operatorname{dog}) \rightarrow t^{\star} \text { (animal) } \\
& t^{\star}(\text { to stab }) \rightarrow t^{\star}(\text { to hurt }) \\
& \text { was killed by } \leftarrow \text { (Caesar, Brutus }) \\
& \text { was killed by } \nleftarrow \text { (Cicero, Brutus) }
\end{aligned}
$$

The next step is the introduction of a new term forming operator $s$, forming event terms from sentences (remember now that 'sentence' stands for 'universal and singular sentence'):

Definition 1. The term $s A$ is an event term if and only if $A$ is a sentence, and

1. all terms in $A$ are empirical terms; and

2. A is neither logically or analytically true nor logically or analytically false.

The first condition is due to the fact that the background is causal logic. If someone is sure that 7 is a prime number or Part of the Good is the belief in Truth and Personal Responsibility constitutes an event it is her/his business

\footnotetext{
${ }^{3}$ The predication theory used is laid out in [15], pp. $176 \mathrm{ff}$.
} 
to drop the condition or to choose a weaker one - such "events" do not play any role in causal relations. A lot of boring events, also standing in no connection with causality, would exist if the second condition were rejected.

It seems to be clear that the conditions are not too strong, but are they too weak? Let us offer further conditions:

Only atomic sentences constitute events.

This condition should and can prevent the existence of tricky and sophisticated events consisting of unconnected parts: the event, for example, that Bush is the president of the USA and Jane is nicer than Tarzan. But especially in social sciences most interesting practical cases of causes and effects are compound and structured and therefore rejected by the condition. Moreover, it is quite difficult to define an interesting "part of"-relation on events only dealing with atomic sentences. ${ }^{4}$

\section{Only true sentences constitute events.}

The idea behind this condition is, of course, the wish to have truth-makers for sentences. If events are truth-makers for sentences (as will turn out to be the case in the current analysis), one could argue as follows: if the sentence is true, there is an event, to which it belongs in some way; but if the sentence is false, there cannot be an appropriate event - if there were one, the sentence would be true. This idea can be used to define existence of events in terms of true sentences; dropping the condition allows for constituting fictive, future, and past events. Because explanation, prediction and other human actions deal with such entities, the condition seems to be too strong.

\section{Only unnegated sentences constitute events.}

There are a lot of difficulties with events of type 'the event, that not...': to which spatiotemporal region they belong? do they have parts? is it possible for them to occur in causal structures? and many others. Part of this paper is concerned with such questions. At the moment it is enough to mention, that it is counterintuitive to exclude all such events (forbearance is something's not occurring and the absence of power in a net is an event in

\footnotetext{
${ }^{4}$ In private discussion I was asked, whether there is an intuitively well-grounded example for an event with an unconnected spatiotemporal region. Consider the event, that Adam was talked into depositing all his money in the United Uncertainty Bank last year and that their Australian partners went into a financial crash last week - I believe that this well may be considered as a single event, which is the cause for Adams bad dreams last night.
} 
the common sense), and not so easy, given a suitable substitution rule in complex event terms.

All event terms obtained by the rule describe event tokens: concrete, singular, spatiotemporally localized events. This is due to the fact, that all sentences refer to a concrete spatiotemporal region (however large it may be), and to concrete individuals or explicitly to some or all individuals of a type. Given these singular event terms, it is easy to construct terms describing event types (general event terms), that means: schemes, types, kinds of events. Both of the following constructions allow us to understand the relation between singular event terms and appropriate general event terms as a relation of meaning inclusion. Furthermore, after introducing general event terms it seems natural to understand singular events as particular instances of universal event schemes. The main idea of the following rules is to weaken the conditions on "sentence":

Definition 2. The event term $s B$ is an event scheme for $s A$ with respect to spatiotemporal regions if and only if $s B$ can be obtained from $s A$ by erasing 0 or more occurrences of spatiotemporal constants and variables (with their quantifiers) in $s A$.

Definition 3. The event term $s B$ is an event scheme for $s A$ with respect to subjects if and only if $s B$ can be obtained from $s A$ by erasing 0 or more occurrences of term quantifiers in $s A$ or by substituting subject terms $\left(u^{a 1}, u^{a 2}, \ldots\right)$ by subject terms $\left(u^{b 1}, u^{b 2}, \ldots\right)$ in $s A$ such, that $t^{\star} u^{a 1} \rightarrow$ $t^{\star} u^{b 1}, t^{\star} u^{a 2} \rightarrow t^{\star} u^{b 2}, \ldots$

Definition 4. The event term $s B$ is an event scheme for $s A$ if and only if it is possible to construct a finite number of event terms $s B^{1}, s B^{2}, \ldots, s B^{n}$, and it holds: $s B^{1}$ is scheme with respect to spatiotemporal regions or with respect to subjects for $s A, s B$ is a scheme with respect to spatiotemporal regions or with respect to subjects for $s B^{n}$, and all neighboring constructed event terms are in one of these relations.

Observe, that there are schemes of schemes, and that the relation of being a scheme for is reflexive, nonsymmetric, and transitive.

Two axioms should be added before speaking about existence:

Terms describing identical events (whatever identity of events is) have the same instances and the same schemes.

If $s B$ is a scheme for $s A$, then $t^{\star} s A \rightarrow t^{\star} s B$. 
The first axiom makes the relation of being a scheme for independent of the concrete linguistic form of the given event terms. The second axiom guarantees the possibility of term quantification over general event terms by extending the relation of meaning inclusion to event terms.

\section{Events}

The next few words are about ontology. What kinds of entities do we need to introduce events into the language? Words (terms), sentences, operators. Also the construction of general event terms (hence: the possibility to quantify over events) is purely linguistic. The weakest claim about what an event is, can be made by defining:

Definition 5. An event is what is described by an event term. In more detail: (i) An event token is what is described by a singular event term. (ii) An event type is what is described by a general event term.

This is, of course, somewhat tricky. Such a definition has, however, one advantage: according to it all further ontological conceptions about what events are, are allowed. If someone uses event terms she/he has to know to which entities they have to be applied.

Here a remark should be made: An event, says the definition, is something that is given by a special term of the form 'the event, that...', where '...' stands for sentences. As I see it, not all events are really given in such a way. A lot of events are constructed with the help of '-ing' forms or even have proper names. I accept all this insofar as it is possible to reconstruct them in the proposed canonical way. I do not believe, that there is an example for an event, for which the canonical construction is in principle impossible. But if there is one, or if a method could be found to introduce events independent of language, the definition is a restriction: such entities are not of the kind, which the paper is about.

A second remark concerns singular event terms. The sentence in a singular event term constitutes a particular: an event token. Being a particular, an event token has a lot of properties and stands in a lot of relations to other particulars, essential and rather incidental ones. It is in principle impossible to include all of them into the constituting sentence, hence from the event term alone it is not possible to get the whole event in all aspects. This may lead to the idea, that singular terms do not describe event tokens, but rather 
aspects of a token, and therefore describe a kind of type. In the present approach on the contrary, a singular event term is the name of an event token with all its particular properties and relations, expressed or unexpressed in the constituting sentence. Using VAN BENTHEM's metaphor: humans use their language in order to cut the continuous movie into manageable slices, but after cutting along some lines, there is a lot of further information on the slides besides the lines of cutting.

Another important question concerns the minimal ontological status of the events introduced. Is it possible to find a criterion for the existence of events and what does it depend on? As mentioned above it is possible, and the criterion depends on truth:

Definition 6. (i) An event token $s A$ exists if and only if the sentence $A$ is true. (ii) An event type $s A$ exists if and only if there is an event token $s B$ for which $s A$ is a scheme and $s B$ exists.

The second part of the definition is quite natural and clear, kinds of events exist if there is at least one particular existing instance. The first part of the definition says that existence of events depends on exactly the same things on which the truth of the constituting sentence depends.

\section{Example 4.}

The event, that the Socialist Party in France lost the 1992 elections, exists, if in France in 1992 there is (exists) a Socialist Party, and there were at that time elections, and the Socialist Party really lost those elections.

The event, that unskilled workers do not find jobs, exists, if there is a time and a place and a concrete unskilled worker, for which it holds: the event, that at that time and place, this worker does not find a job, exists.

Obviously existing events could play the role of truth-makers: A sentence is true, if it constitutes an existing event follows from definition 6 . The existence of events is not independent, neither of language, nor of the given object ontology. Not only the expressive power of languages determines, which events exist, but some logical features do this as well. Sentences, constituting existing events in a language, may for example turn out to be analytic in another language and therefore not to constitute events at all. The dependence on object ontology is evident because of the dependence of truth on the existence of certain things. 
It is still possible to understand singular events and schemes as being pure linguistic constructions, singular events existing in addition to empirical things as particulars within object ontology and schemes being abstract entities, or even tokens and types of events existing in the empirical world. This can be done by simple changes in definition 6 , giving formally the same results but strengthening the "ontological input" concerning tokens and types. What is not open are two consequences of the whole approach:

1. Events require for their existence true sentences (that is: minimally the existence of certain empirical things).

2. If there is an ontological hierarchy between events, the tokens are prior to the types.

We have now three assumptions:

Event terms are the results of applying a term forming operator to singular and universal sentences or of the construction of schemes from event terms.

An event is what is described by an event term.

An event exists (in the sense you like), only if the constituting sentence is true.

How many events are there?

\section{Parts}

Almost all events can be divided into parts. Particular eruptions of volcanos, sinkings of ships, or the financial crisis of 1929 are complex entities, consisting of other, smaller events. Simons gives in his [11] examples of typical wholes and parts, among them also events: a battle and it's opening shot, an insect's life and it's larval stage ([11], p. 10). Parts of such complex events are often connected causally, but not all of the parts stand in direct causal connections to each other. There is rather a causal story of the form 'there were some starting events, and then because of them this happened and then this, and after some time there was the next event ...' which can be told in order to name the same whole event.

\section{Example 5.}

Take a typical event, the sinking of the Titanic. After the collision with the iceberg it starts: Water comes through the leak, the pumps are 
working without success, water fills the whole ship, and after a time the Titanic is under the water level. Moreover, the sinking of the Titanic is usually also: the passengers' leaving the ship, their struggle to survive in the cold water, the band's playing until the musicians are dead.

Social sciences normally deal with much more complex events, consisting of many interconnected and unconnected smaller events. Interestingly enough, sometimes natural language does not provide clear intuitions about whether an event belongs (as a part in the sense of part of the causal story) to an event or whether it is an effect of it. ${ }^{5}$

If reductionism is right - and it is obviously right to a certain degree - it is possible to tell causal stories about psychological and biological behavior in order to describe social events, to use physical and chemical stories to name biological events, to reduce complex events to their less complex parts. Reduction, therefore, produces lots of events existing with the same (or even prior?) ontological status as the complex events.

How many such parts an event has, depends on the event itself, and on how far the partition makes sense. Maybe, there is a natural end: The description of the behavior of subatomic particles provides us with atomic parts of the event. The following partition provides us with infinitely many parts:

Events often have spatiotemporal parts of the same kind. In the proposed approach not only changes are events, events may be permanent in time (and space). In this case it is possible to construct smaller events by using the same constituting sentence but smaller spatiotemporal regions. Since usually physical time is supposed to be continuous, for a single persistent event there are infinitely many parts, similar to it and to each other and differring only in temporal aspects. An analogous partition produces spatial parts of an event.

\section{Example 6.}

The event, that the Socialist Party rules France in March of 1991, is part of the event, that the Socialist Party rules France in 1991.

The event, that the French government gets taxes from Paris, is part of the event, that the French government gets taxes from France.

\footnotetext{
${ }^{5}$ Is, for example, people's leaving the Titanic an effect or a part of the sinking of the Titanic? It depends, I believe, on whether we are interested in ship building - which pumps are necessary to avoid such a case in the future - , or in the tragic history of Mr. Smith, the passenger of cabin 12 .
} 
Both of the described part relations can be defined with the help of conjunctions:

Definition 7. The event $s B$ is part of the event $s A$ if and only if $s A$ is the same event as $s\left(A_{1} \wedge A_{2} \wedge \ldots \wedge A_{n}\right)$ and $s B$ is $A_{i}, 1 \leqslant i \leqslant n$.

From 6 and 7 follows:

Lemma 1. An event exists if and only if all of its parts exist.

It is worth remarking that this straightforward understanding of a part allows us to solve a nice problem. Let $s A$ and $s(A \wedge B)$ be particular events at the same spatiotemporal region. What about the relation between these events? They are the same, if $s B$ is a part of $s A$ (and this depends on the possibility of a certain description of $s A$ ) and different in other cases. This is the solution to a problem which may - in analogy to "overdetermination" - be called overdescription. It arises if event tokens are supplemented with further specifications, not necessary for their identification.

\section{Example 7.}

Consider Elspeth's quickly eating all the brownies in the refrigerator under the deck at noon ${ }^{6}$. Is the event, that Elspeth ate all the brownies in the refrigerator under the deck at noon, the same event, as the event, that Elspeth ate all the brownies in the refrigerator under the deck at noon and did it quickly? Since Elspeth's eating was quick by assumption, the first event can be described in a way that includes the second part of the second event as a part. Therefore the events are the same. The event, that Elspeth ate all the brownies in the refrigerator under the deck at noon and Adam watched TV, differs from the first event mentioned above.

\section{Fictions}

The existence of events is a "timeless" existence: due to the sentences used if an event exists, it exists in all eternity. It is useful to define a more intuitive notion of existence at some spatiotemporal region, which captures the intuitive understanding. The required property is that of being realized:

\footnotetext{
${ }^{6}$ The example is taken from [2].
} 
Definition 8. (i) Let the event token $s A$ exist. Then it is realized at any spatiotemporal region, which includes some part of the spatiotemporal region, to which the event constituting sentence refers. (ii) An event type is realized at any spatiotemporal region, at which its instances are realized.

This definition provides the opportunity to introduce kinds of actually nonexisting events:

Definition 9. (i) An event is a fiction at a spatiotemporal region if and only if it is at this region an unrealized instance of an existing event. (ii) An event is a pure fiction if and only if it is unrealized at all spatiotemporal regions.

It follows:

Lemma 2. A pure fiction is an instance only of nonexisting events.

Definition 10. (i) An event is at a spatiotemporal region a past event, if it is unrealized at this region and all events which it instantiates have a realized instance at an earlier spatiotemporal region. (ii) An event is at a spatiotemporal region a future event, if it is unrealized at this region and all events which it instantiates have a realized instance at a later spatiotemporal region.

\section{Example 8.}

The event, that Caesar killed Brutus, is a fiction. It is an unrealized instance of the event, that a man killed a man.

The event, that Adam lives on the sun, is a pure fiction with respect to the predicate 'lives on the sun'. It is an unrealized instance of an nonexisting event.

The event, that Brutus killed Caesar, is a past event at present and a future event 10 days before he actually did it.

In some sense future events are also a kind of fiction: often we do not know, whether a sentence which refers to a future spatiotemporal region is true or not. Sometimes it turns out to depend on our own actions, whether a certain sentence becomes true - we may change the future. Is it possible to alter the past? Altering the past would consist in changing the truth-value of an event constituting sentence. URCHS gives an example of how this could be done: 
Think e.g. about a successful fake in History. (I am sure you are not going to ask for an example, of there are none - any perceived fake was definitely not a successful one.) The future moment, at which the present action will have caused a change in the past depends on how long the additional effects of the acting will need to expire. (This is necessary to avoid the possibility of back tracing, which would allow for discovering the intervention - that is the fake.) ([12])

What changes is rather our knowledge about the past, than the past itself. It is not so hard to find such a successful fake in History, if we are allowed to restrict the case to only some people: Even today a lot of fans of Sherlock Holmes - and not only children - mail letters to Baker Street, London. For them the fight at the Reichenbach Falls is undoubted, there and then Holmes almost lost his life. But, in order to make the event existent, that Holmes fought with two Gangsters, Holmes, and his stick, and the Gangsters, and Dr. Watson should exist too! That is the point, where ontology comes in: changing the past, in this sense, requires to make existent things nonexistent and vice versa.

\section{Negative events}

Thus far the paper has dealt with very many and, depending on ontological liberality, quite strange events. The topic changes to really shadowy events by using negation: there are existing negative events. Are there?

Because we are concerned with philosophy, the question has to be settled in two directions. On the one hand we want to know, whether negative events exist, given our definitions; on the other we are interested in solving the question: is it generally appropriate to have such events, or should we exclude them. The following is an attempt to define the intuitive notion of a negative event:

Definition 11. The event $s A$ is negative if and only if the constituting sentence $A$ is either a sentence with negation as main operator or a negative predicative statement (in the sense of denying a property of an object).

Clearly true sentences of the required type are quite common, hence negative events exist. Here are some examples for them:

\section{Example 9.}

The event, that Adam was not on time today; the event, that Brutus did not strangle Caesar; the event, that it is not the case, that Adam 
is in trouble and his wife is happy about it; the event, that Brutus did not stab Caesar in 1914

In fact, using a standard meaning of "being late" and "being on time", the sentence Adam was not on time today is true if and only if the sentence Adam was late today is true. This is tantamount to: The event, that Adam was not on time today exists, if and only if The event, that Adam was late today exists (by definition 6). A similar consideration concerning the logical constants shows, that The event, that it is not the case, that Adam is in trouble and his wife is happy about it exists, if and only if The event, that Adam is not in trouble or his wife is not happy about his trouble exists. Thus, meaning postulates and logical rules link the existence of (some) positive and negative events.

Definition 11 guarantees not only the existence of negative events, having positive "counterparts", but also of many others. It should be mentioned, however, that the restrictions on sentences according to definition 1 are valid for definition 11 , too. ${ }^{7}$

The second part of the question is a normative problem: should there be negative events? I think there should. This needs an explanation, because otherwise some of the provided definitions should be changed. The following summarizes some arguments in favor of and against negative events:

A first intuitive argument against negative events is, that if there are any, there are too many of them. Suppose a very simple structure: a subject term, a predicate term, the corresponding sentence which claims that the object has the property at a certain spatiotemporal region. Suppose further, that the sentence is true and that the object has the property only at this spatiotemporal region. Now it is easy to construct numerous negative events, simply by negating sentences, which assert the wrong property or the wrong spatiotemporal region: There is the event that it is not true that the object does not have the property at the given region, and there are all its parts; there are all the events that the existing object does not have the property at any other region; and there are of course all the events that the not yet or no longer existing event does not have the property in question. Since

7 RusSELL was obviously convinced, that the existence of negative facts is more than a matter of definition. Even though he would not have accepted the definition 11 ("There is no formal test"), he was sure about the metaphysical importance of such entities: "It seems to me that the business of metaphysics is to describe the world, and it is in my opinion a real definite question whether in a complete description of the world you would have to mention negative facts or not." ([9], p. 215). 
usually we deal with continuous and infinite models of spacetime, there are obviously uncountable many such events.

\section{Example 10.}

The event that Brutus killed Caesar on the Ides of March in Rome (in 44 B. C.) - this event guarantees the existence of the following negative events:

The event that it is not the case, that Brutus did not kill Caesar at the Ides of March in Rome (in 44 B. C.)

The event that Brutus did not kill Caesar on the Ides of May in Rome (in 44 B. C.)

The event that Brutus did not kill Caesar on the Ides of March in Alexandria (in 44 B. C.)

The event that Brutus did not kill Caesar in 1914

and others.

This amounts to arriving at uncountably many negative events, given the continuous spatiotemporal structure mentioned above. Using the mentioned analogy, one could say: "Experience is not only the continuous movie on the screen, it consists also of the negations of all the not-shown movies".

A second, more philosophical argument against negative events consists in the observation, that negative events by themselves are often unspecific. Frequently a negative event leaves it open what really happened, while positive events are concrete:

\section{Example 11.}

Consider the events from example 10. Except the first of the negative events all of them are really "shadowy": Of course, neither on the Ides of May, nor in Alexandria,..., did Brutus stab Caesar, but what happened instead?

An interesting proposal concerning the unspecificity of negative events was made in [4], and $[5]^{8}$. Negative events are epistemically dependent on their unnegated counterparts. They play a role in human ontology only in cases, in which people are afraid of, wait for, believe in the probability or possibility of ... these counterparts. These remarks are an argument for linguistic conventionalism, according to which event terms are used to cut slides from the movie, from empirical world.

\footnotetext{
8 The term 'shadowy entity' is taken from these papers.
} 
Formally the suggested idea has to be realized in form of restrictions on the existence of negative events. Let us use the proposed definitions about existence of events only for undoubtly positive events and then state:

Definition 12. (i) If there are identical events and one of them exists, all of them exist. (ii) If $s A$ is a negative event, $s A$ exists only if the sentence $A$ is true and there is a person epistemically involved in the positive counterpart of $s A$.

The first sentence precludes, that positive events under a negative description fall under the restriction. It says that if there is a positive formulation, it should be used in ontology. The definition is not quite clear because of the unknown person and the uncertain epistemic involvement. The next few words about why negative events are necessary should clarify at least the direction of interpretation.

Event terms describing negative events are not only part of philosophical terminology but also of common language and the language of theoretically reflected practical behavior: in action theory the decisive requirement is intentionality and without doubt someone can intentionally let things go their way. There, may be, was not the action of changing the course, but the event, that the person did not changed the course, occurred. Moral judgements are made not only about what was done, but also about what was not done in a situation. In an epistemic sense it is sometimes more interesting to know what did not happen than what happened. All this shows that negative events are embedded in a causal structure, they are able to be subjects in causal statements. Some of these causal statements are practically important, allowing us to prevent the future occurrence of events. If the existence of negative events depends in such a way on human aims and purposes, it is not surprising that strange and useless events can be construed by neglecting the epistemic connections. Nevertheless, it is a reasonable question to ask, whether there is an identical positive event for any negative event or not. This depends on the language used: it is easy to imagine languages, not differing in any respect except the existence of such an identical positive event for a given negative one.

A straightforward solution to the problem consists in restricting the existence of all, or at least all negative events without positive counterpart, to existence in an epistemic system. This can be done by introducing a parameter: ${ }^{9}$

\footnotetext{
${ }^{9}$ Another conception of events, similar to the proposed one with respect to the intro-
} 
Definition 13. An event $s A$ exists for a set $\mathcal{M}$ of epistemic subjects, if and only if $s A$ exists in the sense of definition 6 , and an $\mathcal{A} \in \mathcal{M}$ is definitely interested in $s A$.

In a well defined sense there are events, types of events, their parts, negative events. They are, because any true predication requires the existence of the subjects of predications. If events are ontological parts of the world in the same right as empirical things (the other kind of particulars), our ontology is overloaded. The conclusion to be drawn was suggested by the introduction of this paper: events are not pure empirical entities. They depend on empirical entities (through the truth of sentences) and on language. Furthermore: they are connected with human wishes, expectations, fears and a lot of other attitudes. Events form another kind of ontology, besides the ontology of things, supervenient on the latter, but not totally determined by it.

\section{References}

[1] M. Brand, Intending and Acting, Cambridge, London 1984.

[2] C. Cleland, "On the Individuation of Events", Synthese 86, 1991, pp. 229-254.

[3] D. Davidson, "The Individuation of Events", [in:] Essays in Honor of Carl G. Hempel, ed. by A. Anderson and others, Dordrecht 1969, pp. 216-234.

[4] H. Geser, "Elemente zu einer soziologischen Theorie des Unterlassens", Kölner Zeitschrift für Soziologie und Sozialpsychologie 38, 1986, pp. 643-669.

[5] H. Geser, "Ueber die wachsende Bedeutung des Unterlassens in der 'Aktiven Gesellschaft'”, Schweizer Zeitschrift für Soziologie, 1986, pp. 71-90.

[6] J. Kim, "Events and Their Descriptions: Some Considerations", [in:] Essays in Honor of Carl G. Hempel, ed. by A. Anderson and others, Dordrecht 1969, pp. $198-215$.

[7] D. Lewis, "Events", [in:] Philosophical Papers, Vol. II, New York, Oxford 1986, pp. 241-269.

[8] L. Lombard, Events A Methaphysical Story, London, Boston, Henley 1986.

[9] B. Russell, "The Philosophy of Logical Atomism", [in:] Logic and Knowledge, London 1964

duction of an epistemic reference but different from it, for example, in introducing still more ontological levels, can be found in [12]. 
[10] A. Sinowjew, H. Wessel, Logische Sprachregeln, Berlin 1975.

[11] P. Simons, Parts A study in Ontology, Oxford 1987.

[12] M. Urchs, "Causal Priority — Towards a logic of event causation" (unpublished manuscript).

[13] J. van Benthem, The Logic of Time, Dordrecht, Boston, London 1983.

[14] P. van Inwagen, An Essay on Free Will, Oxford 1983.

[15] H. Wessel, Logik, Berlin 1984.

Uwe SCHEFfler

Institute of Philosophy

Humboldt University

Unter den Linden 6

D 10099 Berlin, Germany 